\title{
Minimally Invasive Plate Osteosynthesis In The Treatment Of Isolated UInar Bone Fractures
}

\author{
Suman K Shrestha, MS, Pramod Devkota*, MS, Laxmi P Mainali**, MS \\ Department of Orthopaedics and Trauma Surgery, Patan Academy of Health Sciences Patan Hospital, \\ Lalitpur, Nepal \\ *Department of Orthopaedic Surgery, Kaski Sewa Hospital, Pokhara, Nepal \\ **Department of Orthopaedic Surgery, Om Sai Pathivara Hospital, Jhapa, Nepal
}

\begin{abstract}
Background: Minimally invasive internal fixation is also called "biological internal fixation". This concept is used widely in the treatment of various fractures but to date, there have been no report about such application in the repair of isolated ulnar bone fractures. Methods: Eleven patients with fracture of isolated ulnar bone (four AO type 22A11 and seven AO type 22A12), mean age 43.16 (range, 24- 59y), were treated using closed reduction and locking compression plate with minimally invasive plate osteosynthesis (MIPO) with the aim of minimising soft tissue damage. Results: Fractures healed at an average of 7.8 weeks with good to excellent clinical outcomes. There were no complications such as nonunion, implant failure or neurovascular injuries. Conclusions: MIPO seems to be advantageous for soft tissue and bone biology. Good union was seen and fracture complications were also prevented by early mobilisation.
\end{abstract}

Key Words:

Locking compression plate (LCP), closed reduction, isolated ulnar bone fracture, minimally invasive plate osteosynthesis (MIPO)

\section{INTRODUCTION}

An isolated fracture of the ulnar shaft is a common injury usually caused by direct trauma. Ulnar shaft fractures have been extensively studied and various treatments such as open reduction and internal fixation, plaster cast immobilisation and a functional brace have been recommended ${ }^{1-5}$. The perception is that isolated ulnar shaft fractures treated by nonsurgical means frequently fail to unite; there are recommendations for internal fixation in preference to cast stabilisation as even the above-elbow cast does not provide sufficient immobilisation to facilitate healing ${ }^{6}$.

The traditional method of open reduction and plate fixation requires wide exposure of the fracture site with stripping of the soft tissues, which may in turn devascularise fracture fragments ${ }^{7}$. This may contribute to necrosis caused by trauma and consequently increase the risks for delayed healing and infection. Open techniques entail a larger incision, more bleeding and a need for periosteum stripping during surgery. Postoperative recovery is also protracted. Minimally invasive plate osteosynthesis (MIPO) have been described for some fractures of lower and upper extremities $^{8,910,11}$. This technique was developed to avoid extensive exposure of the fracture site and to minimise soft tissue damage, and entails a smaller incision resulting in a smaller scar and recovery of the soft tissue occurs more rapidly. To date, there are no reports of adaptations of this approach for fractures of the isolated ulna. The ulna is a subcutaneous bone, and it is relatively easy to fix fractures of the ulnar shaft using the MIPO technique.

The purpose of this prospective study was to report on the MIPO technique and clinical outcomes of such percutaneous medial plate stabilisation of isolated ulnar bone fractures. We hypothesised that MIPO is a safe and effective option for the treatment of this fracture.

\section{MATERIALS AND METHODS}

From June 2008 to September 2010, eleven patients who sustained closed displaced fractures of the isolated ulna were enrolled in this study after giving informed consent and obtaining approval from the hospital ethical committee (Figure 1). All patients at the Department of Orthopaedics and Trauma Surgery of Patan Hospital during this time period were treated with of MIPO with a $3.5 \mathrm{AO}$ locking compression plate (AO LCP).

The cause of injury was direct hit in three patients, and fall and road traffic accident in four patients each. In four cases, the fracture type was $22 \mathrm{~A} 11$, and in seven cases $22 \mathrm{~A} 12$. The mean follow up period was 19 months (range, $12-29 \mathrm{~m}$ ). The age of patients ranged from 24 to $59 \mathrm{y}$ (mean, 43.16y); there were eight male and three female patients, six of whom had injured their dominant forearm. Five patients had fractures at the distal third of the forearm and the other six 
fractures were at the proximal third of the forearm. All patients were seen within five days of injury and operative procedures were performed at a mean of eight days (range, 5-11days) following fracture. Patients who sustained bilateral forearm fractures or isolated ulnar bone with distal radio-ulna joint dislocations were excluded from this study. All analyses were conducted using Microsoft Excel for Windows.

Anteroposterior (AP) and lateral radiographic views of the affected forearm were obtained in all cases. Preoperative planning included tracing an implant template on the AP view of the forearm.

Patients were placed supine on the radiolucent operating table under regional or general anaesthesia and a tourniquet was applied at the arm. The shoulder joint was positioned at $90^{\circ}$ abduction, $90^{\circ}$ flexion at the elbow and a pronated forearm. Fluoroscopic views of the entire forearm including the elbow and wrist (AP, lateral, oblique views) joints were available. Two approximately $2-\mathrm{cm}$ long incisions were made proximal and distal to the fracture site between the extensor carpi ulnaris (ECU) and flexor carpi ulnaris (FCU). Dissection was then carried out deep towards the bone, properly visualising the anatomical structures, and dissecting distal to proximal on the medial side of ulna. A subcutaneous extra-periosteal tunnel was prepared between the ECU and FCU and the underlying periosteum with a narrow periosteal elevator inserted first from the proximal incision distally and then from the distal incision proximally (Figure $2 \mathrm{a}$ and $2 \mathrm{~b}$ ). One assistant held the elbow and the other assistant held the wrist joint, while reduction was accomplished while administering traction and counter-traction. Fracture was reduced under close observation via $\mathrm{C}$-arm fluoroscopy and when good reduction was achieved, it was temporarily stabilised with two or three $1.5 \mathrm{~mm}$ Kirschner $(\mathrm{K})$ wires. The plate (7-9 holes, 3.5 AO locking compression plate (LCP); Synthes, India) was inserted through the tunnel on the medial side gliding from distal towards proximal. The plate was mounted on the medial side of ulna subcutaneously. Initially the plate was fixed on the proximal and distal most locking head unit using a bicortical locking head screws (LHS). Two more screws were applied via stab incisions. The third conventional screw was used to reduce malalignment of the fracture as described in principle of MIPO ${ }^{12}$. Lastly, the plate was fixed by three screws on either side of the fracture; all screws were fixed bicortically (Figure 2).

Active finger movements were encouraged immediately after surgery and no splint was applied postoperatively. On the third postoperative day, patients were discharged after inspecting wounds and a post-operative radiograph. Wrist and elbow mobilisation was started under the supervision of trained physiotherapist; sutures were removed on the 14th postoperative day in the out patient department. Patients were followed-up every month for the first four months and then every two months for at least one year (Figure 3).

\section{RESULTS}

The tourniquet time for the surgical procedure ranged from forty minutes to 90 minutes with average of 55 minutes. The final follow up examinations took place at an average of $19 \mathrm{~m}$ (range, $12-29 \mathrm{~m}$ ) after surgery. Fracture healing was defined as callus bridging of one cortex, seen on both lateral and posterior-anterior view radiographs with no tenderness or pain at the fracture site ${ }^{13}$. The average time to union was 7.8 weeks and no major complications such nonunion, delayed union, malunion, angulation at the fracture, deep infection or compartment syndrome were seen. All fractures united in almost absolute anatomical position. Supination and pronation of the injured forearm was almost equal when compared to the normal contralateral side at the one year follow-up. Range of motion of the wrist and elbow were almost equivalent to the normal side. There was no loss of grip strength of the affected hand or forearm muscle wasting. One patient sustained superficial infection at the incision sites which healed uneventfully with regular dressing and oral antibiotics. There was no nerve injury, tourniquet palsy or failure of reduction.

\section{DISCUSSION}

Isolated fractures of the ulnar shaft, a common forearm injury often referred to as a nightstick fracture, usually results from a low energy injury. Routine internal fixation, however, may be associated with complications such as nonunion, delayed union, infection, limitation of motion, refracture of the bone following removal of the plate, synostosis, or peripheral nerve injury ${ }^{14,15,16}$. Unstable fractures are those that are displaced more than $50 \%$,

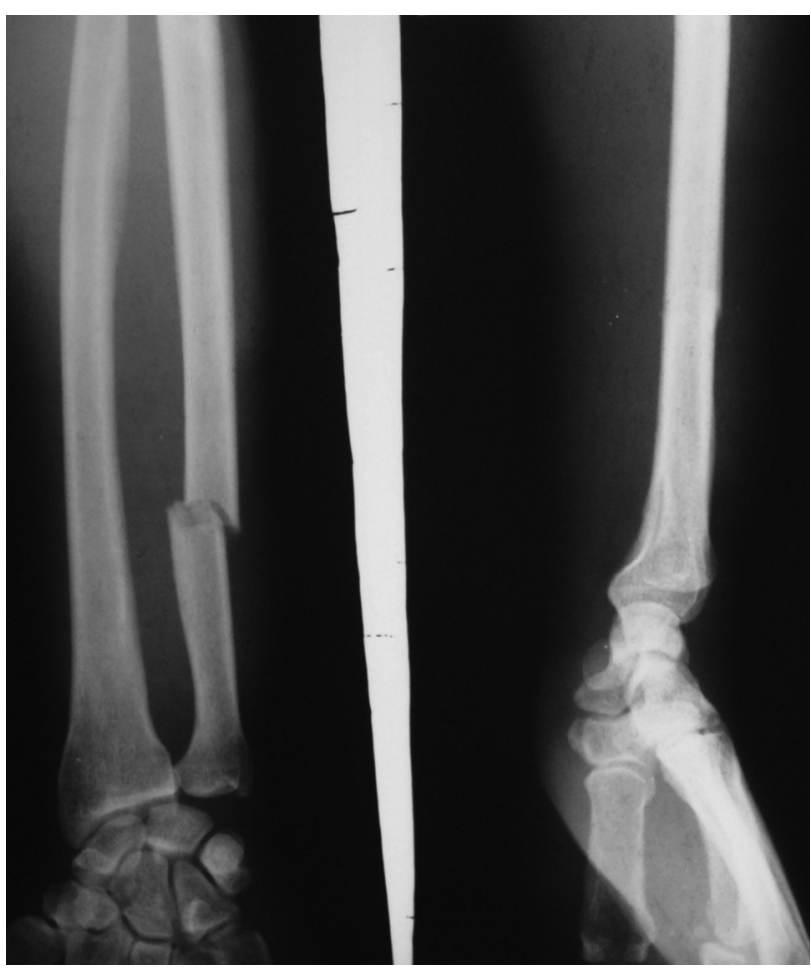

Fig. 1: AP and lateral radiographs of closed isolated ulnar fractures in 49 year old man. 


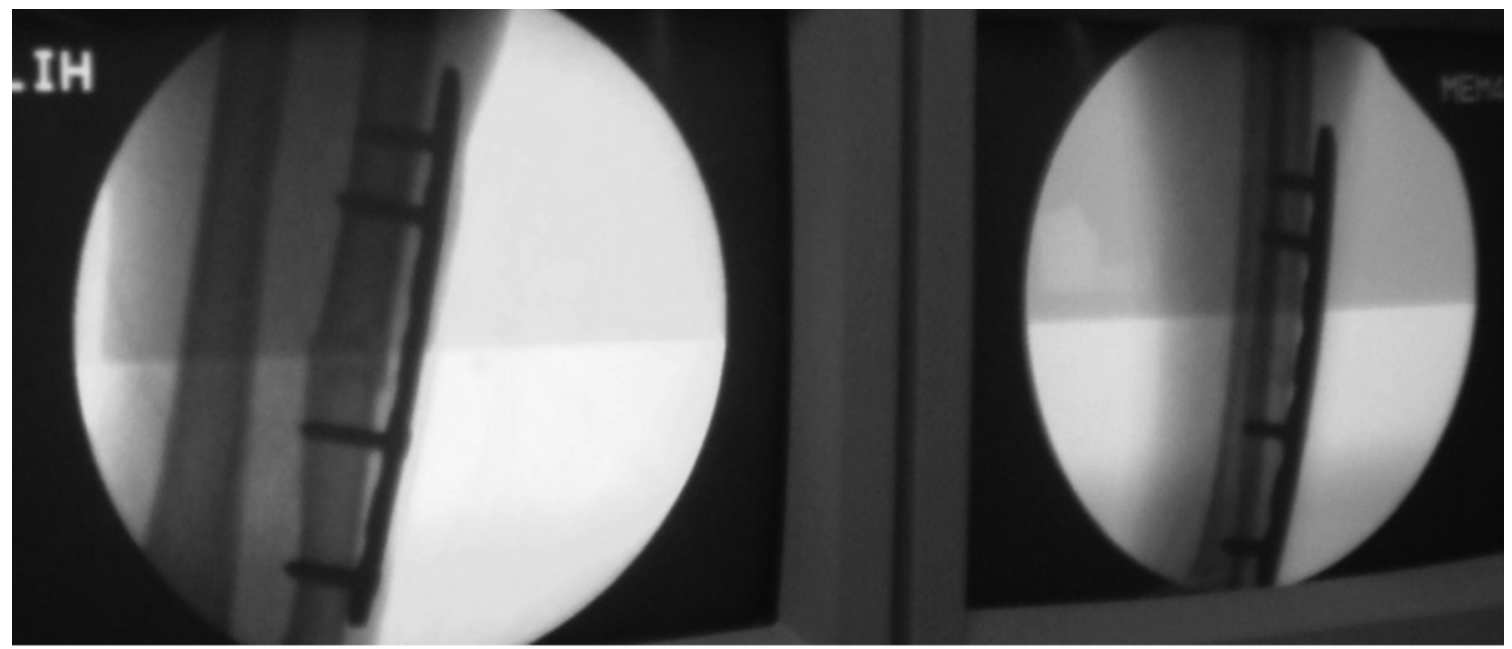

Fig. 2: Intraoperative radiograph while fixing plate through two small incisions C-arm picture.

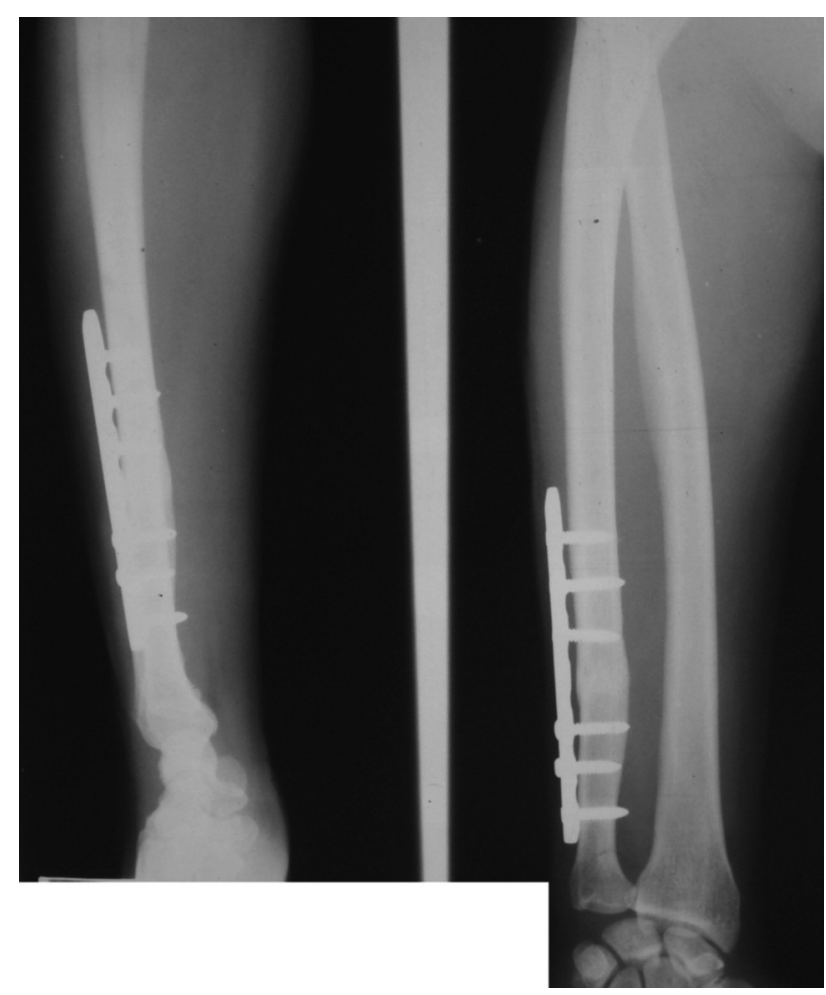

Fig. 3: AP and lateral radiographs after four months, fracture consolidated nicely.

angulated more than $10^{\circ}$, or are located in the proximal third of the ulna ${ }^{17}$. Stable fractures are managed well with forearm bracing ${ }^{1}$. Unstable fractures are reliably treated with open reduction and internal fixation with compression plating. The question of the best treatment regime for isolated ulnar fractures has been addressed by a large number of authors in recent years ${ }^{4,18}$.
Brakenbury noted an increased non-union rate in the fractures located at the distal third of the ulna, where circulation may be compromised due to the lack of muscle coverage and termination of the nutrient artery proximal to this site ${ }^{14}$. In the present series, most patients sustained fractures at the distal third of the ulna, indicating a need for surgical intervention. Stern suggested that the high rate of ulnar non-union in forearm fractures might be due to torsional stresses applied to a relatively stationary ulna during pronation and supination of the forearm ${ }^{19}$.

In the present study, the average union rate was 7.8 weeks, supination and pronation of the forearm was excellent and there was no elbow and wrist stiffness at the last follow-up. Handoll studied the effects of various forms of treatment for isolated fractures of the ulnar shaft in adults and found insufficient evidence from randomised trials to determine which method of treatment is the most appropriate ${ }^{20}$. There was, however, no control group included in this study with which to compare these results and the number of patients was relatively small. We realise that this study of only eleven isolated ulnar shaft fractures treated with this method has limited implications. Large meta-analysis series and randomised control design studies are needed to prove the superiority of MIPO in isolated ulnar shaft fractures.

\section{CONCLUSION}

In this small series, MIPO was more advantageous for soft tissue, bone biology, supination and pronation motion of forearm results. Good union was seen and fracture complications were also prevented by early mobilisation. In isolated ulnar bone fracture, MIPO seems to be a safe and effective surgical treatment method and could be a viable alternative to other techniques. 


\section{REFERENCES}

1. De Boeck H, Haentjens P, Handelberg F, Casteleyn PP, Opdecam P. Treatment of isolated ulna shaft fracture with below elbow plaster cast. A prospective study. Acta Orthop Trauma Surg 1996; 115: 316-20.

2. Dymond IW. The treatment of isolated fracture of distal ulna. J Bone Joint Surg Br 1984; 66: 408-10.

3. Ekelund AL, Nilsson OS. Early mobilization of isolated ulnar shaft fractures. Acta Orthop Scan 1989; 60: 261-2.

4. Gebuhr P, Holmich P, Orsnes T, Soelberg M, Krasheninnikoff M, Kjersgaard AG. Isolated ulnar shaft fractures. Comparision of treatment by a functional brace and long arm cast. J Bone Joint Surg Br 1992; 74: 757-9.

5. Goel SC, Raj KB, Srivastava TP. Isolated fractures of the ulnar shaft. Injury 1991; 22: 212-4.

6. Watson-Jones R. Inadequate immobilization and non-union fractures. Br Med J 1934; 936-40.

7. Heim U, Pfeiffer KM. Internal fixation of small fractures, 3rd Edn. Berlin: Springer, Verlag; 1987.

8. Redfern DJ, Syed SU, Davies SJ. Fractures of the distal tibia: minimally invasive plate osteosynthesis. Injury 2004;35:615-20.

9. Krettek C, Müller M, Miclau T. Evolution of Minimally Invasive Plate Osteosynthesis (MIPO) in the femur. Injury 2002; 33: 460-5.

10. Imatani J, Noda T, Morito Y, Sato T, Hashizume H, Inoue H. Minimally invasive plate osteosynthesis for comminuted fractures of the metaphysis of the radius. J Hand Surg Br 2005; 30: 220-5.

11. Lau TW, Leung F, Chan CF, Chow SP. Minimally invasive plate osteosynthesis in the treatment of proximal humeral fracture. Int Orthop 2007; 31: 657-64.

12. Gautier E, Sommer C. Guidelines for the clinical application of the LCP. Injury 2003; 34: 63-76.

13. Hasenboehler E, Rikli D, Babst R. Locking compression plate with minimally invasive plate osteosynthesis in diaphyseal and distal tibial fractures: A retrospective study of 32 patients. Injury 2007; 38: 365-70.

14. Brakenbury PH, Corea JR, Blakenmore ME. Nonunion of isolated fractures of the ulnar shaft in adults. Injury 1981; 12: 371-5.

15. Hidaka S, Gustilo RB. Refracture of bones of the forearm after plate removal. J Bone Joint Surg Am 1984; 66: 1241-3.

16. Mih AD, Cooney WP, Idler RS, Lewallen DG. Long-term follow-up of forearm bone diaphyseal plating. Clin Orthop Relat Res 1994; 299: 256-8.

17. Sauder DJ, Athwal GS. Management of isolated ulnar shaft fractures. Hand Clin 2007; 23: 179-84.

18. Boussouga M, Bousselmame N, Lazrek K. Surgical management of isolated fractures of the ulnar shaft. Acta Orthop Belg 2002; 68: $343-7$.

19. Stern PJ, Drury WJ. Complications of plate fixation of forearm fractures. Clin Orthop Relat Res 1983; 175: 25-9.

20. Handoll HH, Pearce PK. Interventions for isolated diaphyseal fractures of the ulna in adults. Cochrane Database Syst Rev 2004; 2: CD000523. 